\title{
THE RADIATION STERILIZATION OF IMIPENEM AND CILASTATIN IN THE SOLID STATE
}

\author{
KAROLINA KILIŃSKA', JUDYTA CIELECKA-PIONTEK', ROBERT SKIBIŃSKI², \\ DARIA SZYMANOWSKA ${ }^{3 *}$, ANDRZEJ MIKLASZEWSKI ${ }^{4}$, WALDEMAR BEDNARSKI ${ }^{5}$ \\ and PRZEMYSŁAW ZALEWSKI ${ }^{1}$
}

\author{
${ }^{1}$ Department of Pharmacognosy, Poznan University of Medical Sciences, \\ Świecickiego 4, 60-781 Poznań, Poland \\ ${ }^{2}$ Department of Medicinal Chemistry, Medical University of Lublin, \\ Jaczewskiego 4, 20-090 Lublin, Poland
}

${ }^{3}$ Department of Biotechnology and Food Microbiology, Poznań University of Life Sciences, Wojska Polskiego 48, 60-627 Poznań, Poland

${ }^{4}$ Institute of Material Science and Engieneering, Poznan University of Technology, Jana Pawła II 24, 60-965 Poznań, Poland

${ }^{5}$ Institute of Molecular Physics, Polish Academy of Sciences, Smoluchowskiego 17, 60-179 Poznań, Poland

\begin{abstract}
One of the best sterilization methods for chemically unstable drugs, such as carbapenems, is irradiation. The chemical and physical properties of radiosterilized imipenem and cilastatin were analyzed using spectrophotometric and chromatographic methods. Unstable free radicals were presented in samples after irradiation; their mean lifetime was $142 \pm 64 \mathrm{~h}$. The antibacterial activity of cefozopran hydrochloride irradiated with a dose of $25 \mathrm{kGy}$ changed for Klebsiella pneumoniae and Escherichia coli. Imipenem and cilastatin in the solid state are not resistant to radiation sterilization; therefore, this method cannot be used for sterilization of this compound.
\end{abstract}

Keywords: imipenem, radiation sterilization, radiostability, Q-TOF, microbiological activity

Imipenem monohydrate (IMP), the first analogue of carbapenem antibiotics, is a semi-synthetic derivative of the natural parent compound - thienamycin, which is produced by the bacterium Streptomyces cattleya. IMP has a broad antibacterial activity spectrum against most clinically important species of bacteria, including Gram-positive, Gramnegative, aerobic, and anaerobic microorganisms. It is successfully used in the treatment of lower respiratory tract infections, intra-abdominal infections, gynecologic infections, urinary tract infections, bone and joint infections, skin infections, bacterial septicemia, and endocarditis (1-5). The mechanism of action of this $\beta$-lactam antibiotic is that it inhibits bacterial wall synthesis as a result of binding to penicillin-binding proteins (6). In the kidneys, IMP is rapidly degraded and inactivated by dehydropeptidase- 1 and loses its properties. Therefore, in clini- cal treatment, IMP is combined with cilastatin sodium - a competitive, reversible, and specific renal enzyme inhibitor - in a $1: 1$ ratio. Cilastatin sodium (CS) has no antimicrobial activity and does not affect the action of imipenem (7). IMP/CS is originally prepared as a sterile powder. After reconstitution and preparation, the solution is administered only via the intravenous route. This form of drug delivery and the nature of the antibiotics require that these compounds are maintained at the highest level of sterility. One of the best sterilization methods of chemically unstable drugs such as carbapenems is irradiation. The possibilities offered by this method seem to be the most advantageous for IMP, which is particularly sensitive to elevated temperature, humidity, and the presence of oxidative factors. The use of radiation sterilization requires detailed radiolytic studies on the impact on phar-

* Corresponding author: e-mail: pzalewski@ump.edu.pl 
maceutical properties and changes in the API structure of the drug (8).

In this study, we investigated the effect of ionizing radiation on IMP/CS in the solid state. A standard recommended dose of irradiation (25 kGy) (9) and higher radiation doses (50-400 kGy) have been applied to understand the process of potential changes in IMP/CS after sterilization.

\section{EXPERIMENTAL}

\section{Standards and reagents}

The IMP that can be used for intravenous infusion is available in preparation of imipenem/cilastatin Kabi (Fresenius Kabi Polska Sp. z o.o.), in the form of a white to slightly yellowish off-white sterile powder mixture. Each vial contains $500 \mathrm{mg}$ of imipenem monohydrate and $500 \mathrm{mg}$ of cilastatin as the sodium salt. The $\mathrm{pH}$ range of the solution ranges from 6.5 to 8.5. The imipenem/cilastatin Kabi is formulated with sodium bicarbonate as an inactive ingredient/buffer. Each vial contains sodium bicar- bonate in an amount corresponding to approximately $1.6 \mathrm{mEq}$ of sodium (approximately $37.5 \mathrm{mg}$ ).

All other chemicals and solvents were obtained from Merck KGaA (Germany) and were of analytical grade. High-quality pure water was prepared using the Millipore purification system (Millipore, Molsheim, France, model Exil SA 67120).

\section{Methods}

Irradiation

IMP/CS samples (5 mg each) were weighted into colorless glass vials and closed with plastic stoppers. Samples were exposed to $\beta$-irradiation in a linear electron accelerator LAE $13 / 9$ (9.96 MeV electron beam and $6.2 \mu \mathrm{A}$ current intensity) at 25 , 50, 100, 200 and $400 \mathrm{kGy}$ dose rates.

\section{Electron paramagnetic resonance (EPR) spec- troscopy}

Detection of free radicals was performed using a Bruker ELEXSYS 500 spectrometer (Bruker, Billerica, MA, USA). The irradiated and non-irradi-

Table 1. MIC values (mg/L) of irradiated IMP/CS samples.

\begin{tabular}{|c|c|c|c|c|}
\hline & \multirow{2}{*}{ Microorganism } & \multicolumn{3}{|c|}{$\mathrm{MIC}(\mathrm{mg} / \mathrm{L})$} \\
\hline & & \multirow{2}{*}{$\frac{\text { 0 kGy }}{4}$} & \multirow{2}{*}{$\frac{25 \mathrm{kGy}}{4}$} & \multirow{2}{*}{$\begin{array}{c}400 \mathrm{kGy} \\
8 \uparrow\end{array}$} \\
\hline 1 & Salmonella enteritidis* & & & \\
\hline 2 & Salmonella enteritidis ATCC 13076 & 2 & 2 & $8 \uparrow$ \\
\hline 3 & Salmonella typhimurium* & 8 & 8 & $32 \uparrow$ \\
\hline 4 & Salmonella typhimurium ATCC 14028 & 8 & 8 & $16 \uparrow$ \\
\hline 5 & Staphylococcus aureus* & 8 & 8 & $16 \uparrow$ \\
\hline 6 & Staphylococcus aureus ATCC 25923 & 8 & 8 & $16 \uparrow$ \\
\hline 7 & Klebsiella pneumonia* & 16 & $32 \uparrow$ & $64 \uparrow$ \\
\hline 8 & Klebsiella pneumonia ATCC 31488 & 8 & $16 \uparrow$ & $32 \uparrow$ \\
\hline 9 & Escherichia coli* & 1 & $2 \uparrow$ & $\mathbf{8} \uparrow$ \\
\hline 10 & Escherichia coli ATCC 25922 & 1 & $2 \uparrow$ & $4 \uparrow$ \\
\hline 11 & Pseudomonas aeruginosa* & 32 & 32 & $64 \uparrow$ \\
\hline 12 & Pseudomonas aeruginosa ATCC 27853 & 16 & 16 & $32 \uparrow$ \\
\hline 13 & Proteus vulgaris* & 4 & 4 & $32 \uparrow$ \\
\hline 14 & Proteus vulgaris ATCC 8427 & 2 & 2 & $32 \uparrow$ \\
\hline 15 & Clostridium butyricum* & 2 & 2 & $16 \uparrow$ \\
\hline 16 & Clostridium butyricum ATCC 860 & 2 & 2 & $8 \uparrow$ \\
\hline 17 & Clostridium pasterianum* & 4 & 4 & $16 \uparrow$ \\
\hline 18 & Clostridium pasterianum ATCC 6013 & 2 & 2 & $\mathbf{8} \uparrow$ \\
\hline 19 & Acinetobacter baumannii ${ }^{*}$ & 32 & 32 & $64 \uparrow$ \\
\hline 20 & Acinetobacter baumannii ATCC 19606 & 32 & 32 & $64 \uparrow$ \\
\hline 21 & Enterobacter aerogenes* & 8 & 8 & $16 \uparrow$ \\
\hline 22 & Enterobacter aerogenes ATCC 13048 & 8 & 8 & $16 \uparrow$ \\
\hline
\end{tabular}


The radiation sterilization of imipenem and cilastatin in the solid state

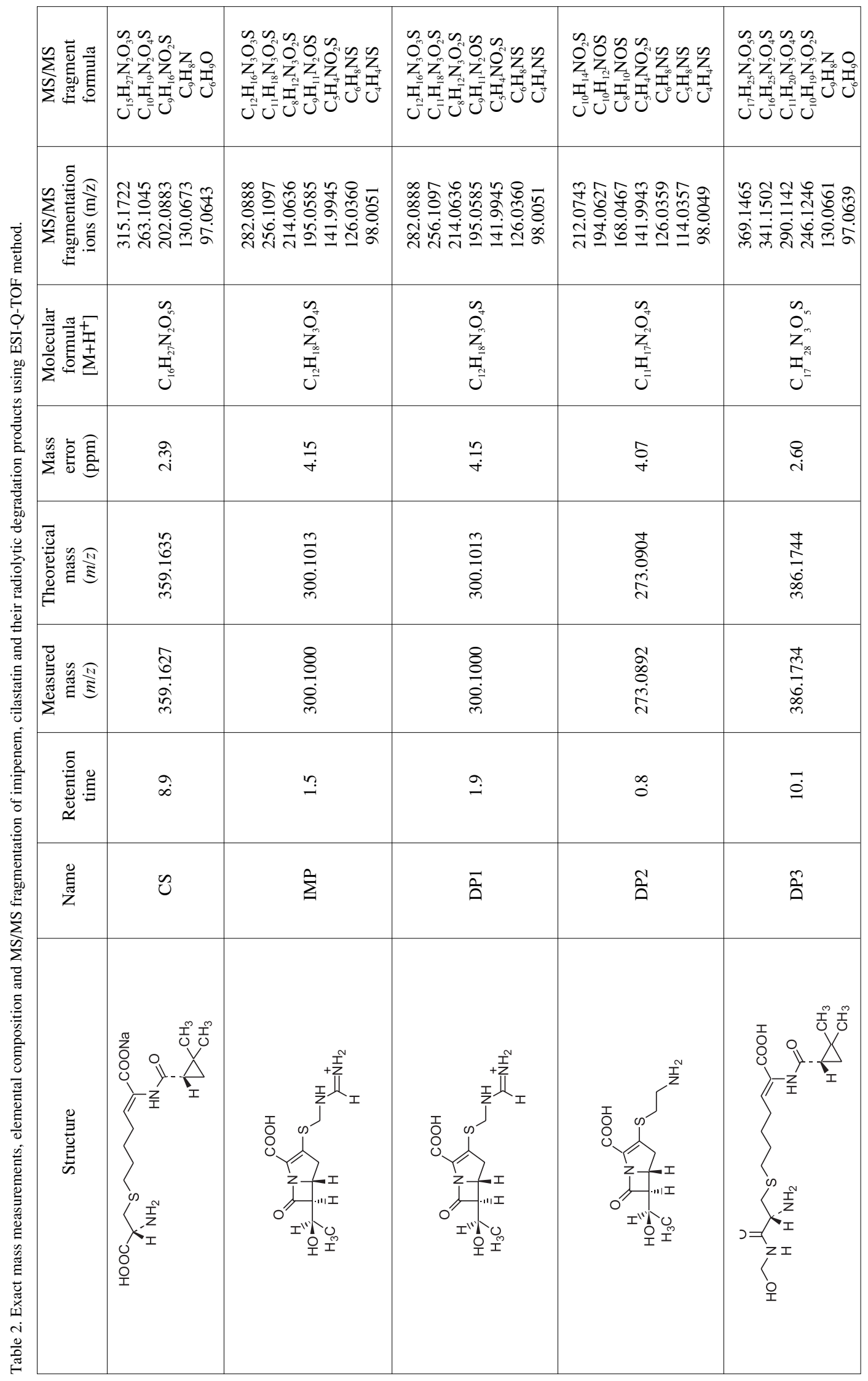


ated powdered samples of IMP/CS were tested in quartz capillaries at a temperature of $24^{\circ} \mathrm{C}$ (X-band, 9.4 GHz). EPR spectra were recorded as a first derivative of the absorption signal. Using the method described by Mai et al. (2013), the concentration of free radicals was calculated (10).

\section{Fourier Transform Infrared spectroscopy}

The spectra were determined using a Fourier Transform Infrared (FT-IR) spectrometer, IR Affinity-1 Shimadzu. Samples were prepared by mixing IMP/CS with potassium bromide in proportions of $1 \mathrm{mg}$ of sample to $300 \mathrm{mg} \mathrm{KBr}$. Pellets were formed under a pressure of $15 \mathrm{ton} / \mathrm{cm}^{2}$ with a barrel having a $13-\mathrm{mm}$ diameter. Absorption spectra were recorded with a resolution of $2 \mathrm{~cm}^{-1}$ within a wavenumber ranging from 4000 to $400 \mathrm{~cm}^{-1}(30$ scans per spectrum).

\section{HPLC-MS/MS analysis}

The analysis was conducted with the use of an Agilent Accurate-Mass Q-TOF LC/MS G6520B

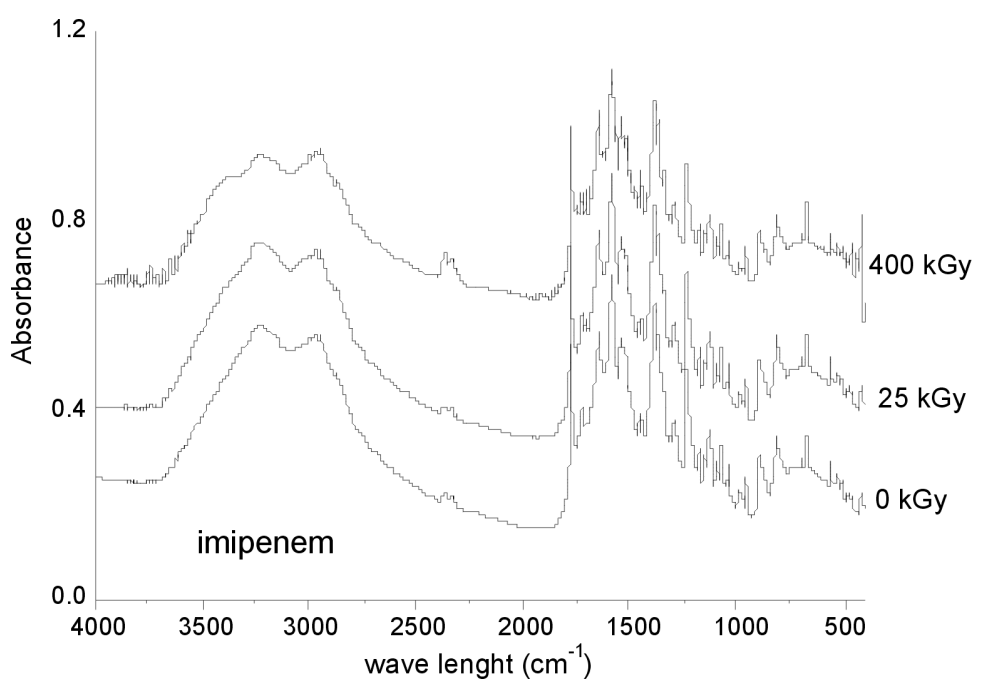

Figure 1. FT-IR spectra of unirradiated and irradiated IMP/CIL

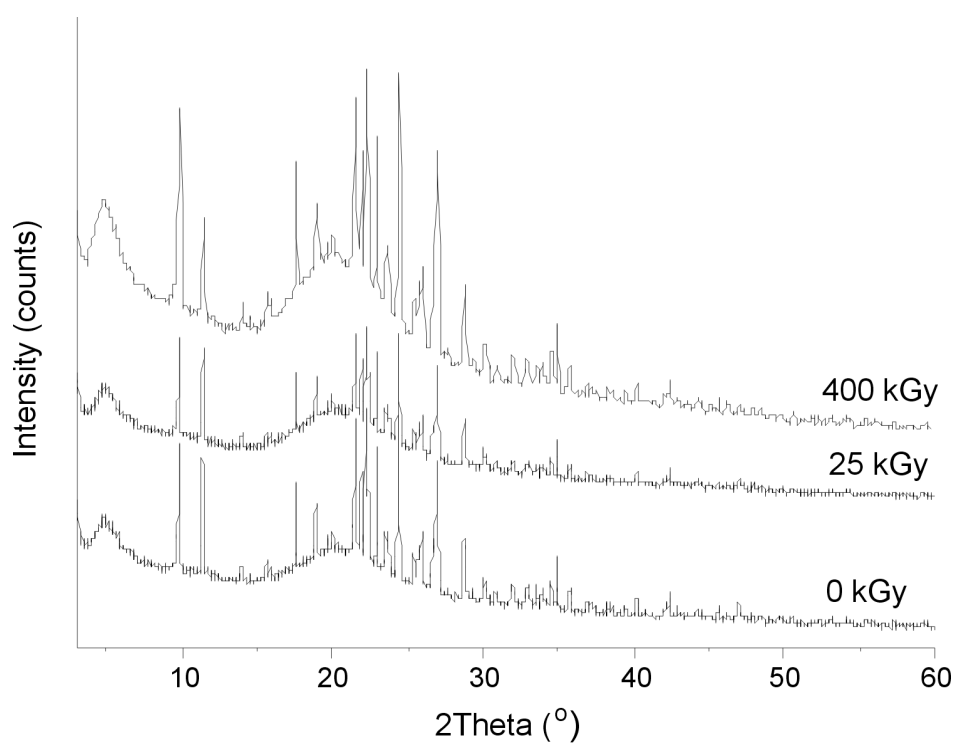

Figure 2. XRPD spectra of unirradiated and irradiated IMP/CS 


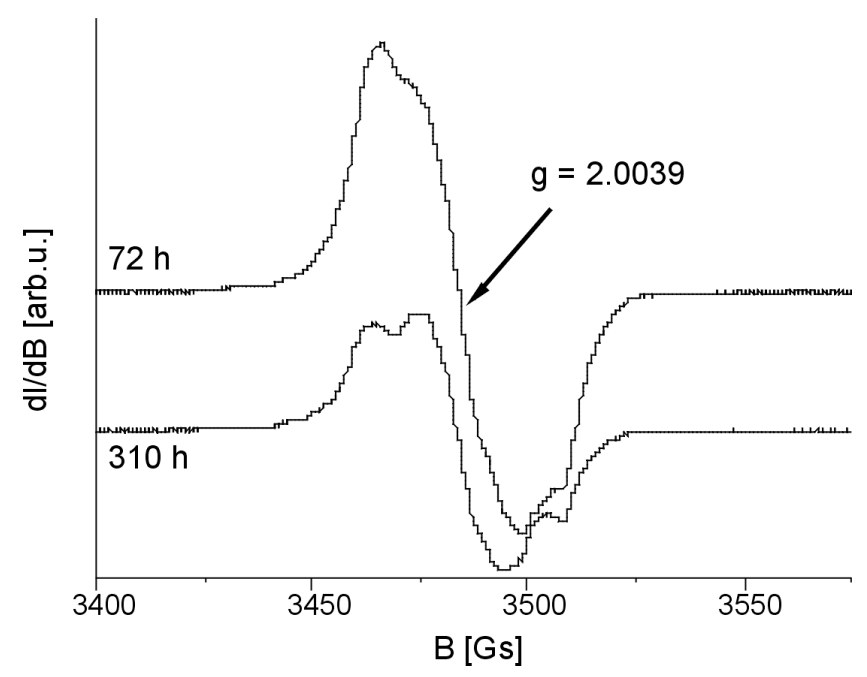

Figure 3. EPR spectra of irradiated IMP/CIL recorded $72 \mathrm{~h}$ and $310 \mathrm{~h}$ after radiation sterilisation (radiation dose $25 \mathrm{kGy}$ )

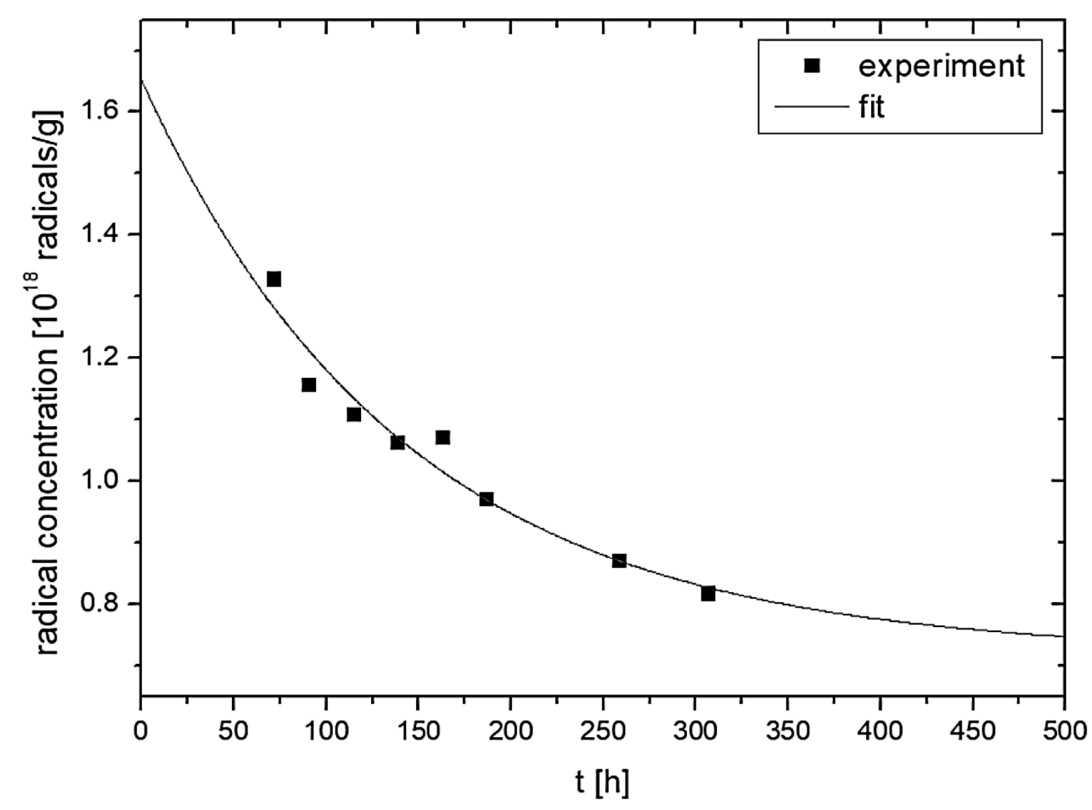

Figure 4. Concentration of free radicals vs. time after radiation sterilisation (radiation dose $25 \mathrm{kGy}$ )

system with a DESI ion source and an Infinity 1290 ultra-high-pressure liquid chromatography system consisting of a G4220A binary pump, a G1330B FC/ALS thermostat, a G4226A autosampler, a G4212A DAD, and a G1316C TCC module (Agilent Technologies, Santa Clara, USA). The control of the system, data acquisition, and qualitative analysis were conducted with the use of the MassHunter workstation software B.04.00. Separations were performed on a Poroshell column $2.7 \mu \mathrm{m}$ particle size, $100 \mathrm{~mm} \times 2.1 \mathrm{~mm}$ (Agilent Technologies, Santa Clara, USA). The initial mobile phase composition was acetonitrile $-0.1 \%$ formic acid $(5: 95, \mathrm{v} / \mathrm{v})$ for $2 \mathrm{~min}$. Then, gradient elution was used, starting from the mobile phase composition ratio $(5: 95, \mathrm{v} / \mathrm{v})$ after 2 min to $30: 70$ within 15 min. The flow rate of the mobile phase was 0.3 $\mathrm{mL} / \mathrm{min}$. The Q-TOF detector was tuned to positive (4 GHz) and the main parameters were optimized as follows: drying gas $10 \mathrm{~L} / \mathrm{min}$, nebulizer pressure 40 
psig, gas temp. $300^{\circ} \mathrm{C}$, capillary voltage $3,500 \mathrm{~V}$, skimmer voltage $65 \mathrm{~V}$, fragmentor voltage $200 \mathrm{~V}$, and octopole 1 radio frequency voltage $250 \mathrm{~V}$. The data were acquired in the auto MS/MS mode with the mass range $\mathrm{m} / \mathrm{z} 50-950$ and the acquisition rate 1.2 spectra/s (for MS and MS/MS data). The collision energy was calculated from the formula $2 \mathrm{~V}$ (slope) $*(m / z) / 100+6 \mathrm{~V}$ (offset) and maximum 2 precursors per cycle were selected with an active exclusion mode after 1 spectrum for $0.2 \mathrm{~min}$. To ensure the accuracy of measurements, the reference mass correction was used and values $\mathrm{m} / \mathrm{z} 121.0508$ and 922.0097 were selected as lock masses.

\section{X-ray powder diffraction}

An X-ray powder diffraction (XRPD) pattern was obtained by using a PANalitycal Empyrean system with $\mathrm{Cu} \mathrm{K}_{\alpha} 1$ radiation (1.54056 A) at a voltage of $45 \mathrm{kV}$ and a current $40 \mathrm{~mA}$. The sample was scanned from $3^{\circ}$ to $50^{\circ} 2 \theta$ using a step size of $0.017^{\circ}$ and a scanning rate of $15 \mathrm{~s} / \mathrm{step}$ with the sample spinning.

\section{Microbiological study}

To verify the antimicrobial activity of IMP, the MIC (Minimal Inhibitory Concentration) values have been determined for each reference strain from the American Type Culture Collection and clinical isolates. The experiments were run in e triplicate. MICs for IMP was assayed using the serial dilutions method on the Mueller-Hinton liquid medium (Merck, Germany), which follows the standards of the Clinical and Laboratory Standards Institute (CLSI) (11). In the tests, microbial culture with a standardized optical density was used.

\section{RESULTS AND DISCUSSION}

Previous research concerning the radiostability of $\beta$-lactams antibiotics has confirmed their susceptibility to degradation (12-17).

Our research on radiostability of imipenem was divided into two areas. The first one is significant due to the application of radiation sterilization and the second one - important from a cognitive point of view, aimed at evaluating the structure of the resulting degradation products.

For IMP samples exposed to irradiation, all changes were investigated using analytical and biological methods. The identity evaluation for irradiated samples was confirmed in the results of spectroscopic (FT-IR), thermal (DCS) and X-ray powder diffraction (XRPD) studies.

At this stage of the study, the MIC of IMP was analyzed against 11 strains of bacteria with patho- genic potential. Both reference strains and clinical isolates were tested. The analysis performed showed the highest MIC value for A. baumannii species (32 $\mathrm{mg} / \mathrm{L}$ ), while the lowest was determined for the $E$. coli species $(1.0 \mathrm{mg} / \mathrm{L})$. Imipenem radiation irradiation with the use of $25 \mathrm{kGy}$ radiation did not lower or increase the MIC value for the tested species. The only two exceptions were $E$. coli and $K$. pneumoni$a e$, which showed an increase in MIC from 1.0 to $2.0 \mathrm{mg} / \mathrm{L}$ and 16 to $32 \mathrm{mg} / \mathrm{L}$ respectively (Table 1 ). It was connected with the formation of one degradation product (DP1) (Table 2). DP1 was a structural isomer of the parent compound. A similar situation was reported for cefozopran hydrochloride after radiation sterilization (15). On the other hand for the $25 \mathrm{kGy}$ irradiation dose, there were no changes in the position and intensity of the characteristic bands of imipenem in FTIR and XRPD (Figs. 1 and 2). It is probably connected with not enough degradation of IMP/CS.

The literature data confirms that the most common problem encountered after radiosterilisation of solid drugs is discoloration or yellowing (17-18). In our study also a colour change of samples after exposure to radiation was reported - from white to slight beige. In the next step, we verified the impact of packing material on irradiated imipenem. None of the tested packages (PVP and glass containers) allowed to obtain sterile IMP/CS without colour changes. What is particularly important, after dissolving the samples of irradiated IMP/CS in water for injections, we get transparent and colorless solutions. Therefore, the authors tend to suggest that the colour change of the IMP/CS in the solid state after exposure to irradiation, may be associated with the presence of the radiolyzed water trapped in the crystal structure of the IMP/CS.

In the EPR spectrum of the non-irradiated IMP/CS no free radical signal is observed, i.e. the concentration of free radicals is less than about $1 \mathrm{ppm}$, which corresponds to the sensitivity of the spectrometer. The spectrum of the irradiated sample (dose $25 \mathrm{kGy}$ ) presented in Figure 3 consists of several lines. The strongest centerline is described by the spectroscopic splitting coefficient of 2.0039 $( \pm 0.005)$. When comparing the spectra presented in Figure 3, which were recorded 72 and $310 \mathrm{~h}$ after irradiation, two conclusions can be drawn. Firstly, the intensity of the spectrum decreases over time, which indicates the presence of unstable free radicals. Secondly, the shape of the spectrum depends on the time that has elapsed since the irradiation, which points out that irradiation of IMP/CS generates at least two different types of free radicals. The 
time changes in the concentration of free radicals after irradiation are shown in Figure 4. The total concentration of free radicals $I(t)$ at any time $t$ after irradiation may be approximated by the exponential function:

$$
I(t)=I_{s}+I_{n} e^{-t / T}
$$

where $I_{s}$ is the concentration of stable free radicals, $I_{n}$ is the concentration of unstable free radicals, and $T$ is the mean lifetime of unstable radicals. The following parameters are obtained by approximations of function (1) to the experimental points presented in Fig. 4: $I(t=0 h)=1.65( \pm 0.22) \times 10^{18}$ radicals $/ g, I_{s}$ $=0.72( \pm 0.12) \times 10^{18}$ radicals $/ g, I_{n}=0.93( \pm 0.10) \times$ $10^{18}$ radicals $/ g, T=142( \pm 64)$ h. In summary, sterilization of the IMP/CS dose of $25 \mathrm{kGy}$ radiation causes radical defects, the concentration of which does not exceed $1000 \mathrm{ppm}$. Stable free radicals account for less than half of all defects generated by irradiation.

In the next part of our research, we evaluated the effect of a higher dose of irradiation (400 kGy). The identity evaluation in the solid state showed changes in the intensity of bands characteristic for molecular domains and changes in the crystallinity of samples. Comparative analysis of FT-IR reveals a change in the spectrum of irradiated IMP/CS (Fig. 1), which corresponds to stretching vibrations $\mathrm{C}=\mathrm{O}$ in a trans-hydroxyethyl group.

In the spectra additional Bragg's reflections appeared, indicating the crystallization tendencies of the IMP/CS, as a result of exposure to irradiation at a dose of $400 \mathrm{kGy}$ (Fig. 2).

Beyond identifying possible physical changes in the structure of irradiated IMP/CS samples, its chemical stability was tested. HPLC-MS/MS was employed for identification of IMP (measured mass 300.1000, retention time $\operatorname{tr}=1.5 \mathrm{~min}$ ) and $\mathrm{CS}$ (measured mass 359.1627, retention time $\operatorname{tr}=8.9$ min) exposed to radiation (Table 2). The molecular ion of IMP and CS were very accurately identified (4.15 ppm and $2.39 \mathrm{ppm}$ ). HPLC-MS/MS analysis has shown three degradation products (measured mass 273.0892, 300.1013 and 386.1744 retention time $\mathrm{tr}=1.5 \mathrm{~min}, 1.9 \mathrm{~min}$ and $10.1 \mathrm{~min})$ of $\mathrm{IMP} / \mathrm{CS}$ after its irradiation (400 kGy) (Table 2). Analysis of the resulting products (Table 2) pays particular attention to the degradation of the imino group. The changes in the antibacterial activity of irradiated IMP/CS (400 kGy) have been reported for all the analyzed stains and increase in the MIC value for all tested microbial strains was observed (Table 1). It should be emphasized that for $\beta$-lactam antibiotics, the substituent in position 3 of $\beta$-lactam ring determines bactericidal activity. Therefore, the authors connect the decrease in the microbiological activity of irradiated IMP (400kGy) against all tested microorganisms with the chemical degradation of the imino group of imipenem.

\section{CONCLUSIONS}

The results of the studies performed, enable us to reach the conclusion that IMP/CS in its solid state is not resistant to ionizing radiation (E-beam) in a standard sterilization dose of $25 \mathrm{kGy}$, and this method cannot safely be used for sterilization and decontamination of this compound. Irradiation with higher doses leads to the following effects: loss in the content of active substance upon irradiation with by about $20 \%$, radiolytic degradation of IMP/CS with 3 products, and decreased microbiological activity for 22 strains.

\section{Acknowledgments}

This study was supported by a grant from the National Science Centre, Poland (Miniatura, 2017/01/X/NZ7/00141).

\section{REFERENCES}

1. Pastel D.A.: Clin. Pharm. 5, 719 (1986).

2. Imipenem/Cilastatin Kabi (package insert). Fresenius Kabi Polska Sp. z o.o. 2012.

3. Kohler T., Michea-Hamzehpour M., Epp S.F., Pechere J.C.: Antimicrob. Agents Chemother. 43, 424 (1999).

4. Barza M.: Ann. Intern. Med. 103, 552 (1985).

5. Kesado T., Hashizume T., Asahi Y.: Antimicrob. Agents Chemother. 17, 912 (1980).

6. Hashizume T., Ishino F., Nakagawa J., Tamaki S., Matsuhashi M.: J. Antibiot. (Tokyo) 37, 394 (1984).

7. Keynan S., Hooper N.M., Felici A., Amicosante G., Turner A.J.: Antimicrob. Agents Chemother. 39, 1629 (1995).

8. Marciniec B., Dettlaff K.: Radiation sterilization of drugs. Trends in radiation sterilization of health care products., p. 187-230, Vienna: IAEA (2008).

9. EN ISO 11137, (2006).

10. Mai V.C., Bednarski W., Borowiak-Sobkowiak B., Wilkaniec B., Samardakiewicz S., Morkunas I.: Phytochemistry 93, 49 (2013).

11. CLSI. M07-A9: Methods for Dilution Antimicrobial Susceptibility Tests for Bacteria That Grow Aerobically; Approved Standard Ninth Edition, USA (2012). 
12. Garbacki P., Zalewski P., Skibiński R., Kozak M., Ratajczak M. et al.: X-Ray Spectrom. 44, 344 (2015).

13. Zalewski P., Szymanowska-Powałowska D., Garbacki P., Paczkowska M., Talaczyńska A. et al.: Acta Pol. Pharm. 72, 1253 (2015).

14. Zalewski P., Skibiński R., SzymanowskaPowałowska D., Piotrowska H., Kozak M. et al.: J. Pharm. Biomed. Anal. 118, 410 (2016).

15. Zalewski P., Skibiński R., SzymanowskaPowałowska D., Piotrowska H., Bednarski W. et al.: Electron. J. Biotechnol. 25, 28 (2017).
16. Kilińska K., Cielecka-Piontek J., Skibiński R., Szymanowska D., Miklaszewski A. et al.: Acta Pol. Pharm. 75, 1127 (2018).

17. Kilińska K., Cielecka-Piontek J., Skibiński R., Szymanowska D., Miklaszewski A. et al.: Molecules 23, 2738 (2018).

18. Ogrodowczyk M., Dettlaff K., Bednarski W., Ćwiertnia B., Stawny M. et al.: Chem. Pap. 72, 349 (2018).

Received: 17.10 .2018 